\title{
Inhibition of aldose reductase ameliorates alcoholic liver disease by activating AMPK and modulating oxidative stress and inflammatory cytokines
}

\author{
CHANGXUAN SHI $^{1 *}$, YUANFANG WANG $^{1 *}$, JING GAO $^{1 *}$, SI CHEN $^{1}$, XIANGQIAN ZHAO $^{1}$, \\ CHENGCHAO CAI $^{1}$, CHANG GUO $^{1,2}$ and LONGXIN QIU ${ }^{1-3}$ \\ ${ }^{1}$ Department of Biological Science and Technology, School of Life Sciences; ${ }^{2}$ Key Laboratory of \\ Preventive Veterinary Medicine and Biotechnology (Longyan University), Fujian Province University; \\ ${ }^{3}$ Fujian Provincial Key Laboratory for the Prevention and Control of Animal Infectious Diseases \\ and Biotechnology, Longyan, Fujian 364012, P.R. China
}

Received May 30, 2016; Accepted April 13, 2017

DOI: $10.3892 / \mathrm{mmr} .2017 .6895$

\begin{abstract}
Aldose reductase (AR) expression is elevated in the livers of patients with alcoholic liver diseases. However, the role of AR in the development of alcoholic liver diseases remains unclear. The aim of the present study was to determine the effect of AR inhibition on ethanol-induced hepatosteatosis in vivo and in vitro, and to identify possible underlying molecular mechanisms. Alcoholic fatty livers were induced in C57BL/6 mice by feeding the mice with Lieber-DeCarli liquid diets. The expression of AR protein was elevated in the liver tissue of C57BL/6 mice fed with an ethanol diet and in mouse AML12 liver cells exposed to ethanol. In addition to the elevation in AR, hepatic steatosis was observed in ethanol diet-fed mice, and this ethanol-induced steatosis was significantly attenuated by inhibiting AR activity with a specific inhibitor, zopolrestat. The suppressive effect of AR inhibition was associated with decreased levels of hepatic lipoperoxides, decreased protein expression of hepatic cytochrome P450 2E1 (CYP2E1), increased phosphorylation of 5'-AMP-activated protein kinase (AMPK) and decreased mRNA expression of tumor necrosis factor- $\alpha$ (TNF- $\alpha$ ). Treatment with the AR inhibitor attenuated the level of lipid accumulation and oxidative stress, activated AMPK, and suppressed the mRNA expression of TNF- $\alpha$, interleukin- 6 and transforming growth
\end{abstract}

Correspondence to: Professor Longxin Qiu, Department of Biological Science and Technology, School of Life Sciences, Longyan University, 1 North Dongxiao Road, Longyan, Fujian 364012, P.R. China

E-mail: qlongxin@tom.com

*Contributed equally

Key words: aldose reductase, alcoholic liver disease, 5'-AMP-activated protein kinase, oxidative stress, inflammatory cytokines factor- $\beta 1$ in ethanol-treated AML12 cells. The results of the present study demonstrated that inhibition of AR ameliorated alcoholic liver disease in vivo and in vitro, in part by activating AMPK, decreasing CYP2E1-mediated oxidative stress and ameliorating the expression of pro-inflammatory cytokines.

\section{Introduction}

Alcohol is a widely-consumed hepatic toxicant, and excessive alcohol consumption may lead to acute and chronic fatty liver diseases, including steatosis, steatohepatitis, liver fibrosis and liver cirrhosis. Although the pathogenesis of alcoholic liver disease (ALD) remains to be fully elucidated, oxidative stress is considered to be one of the principal factors responsible for alcoholic liver damage (1). Previous studies have demonstrated that ethanol-induced oxidative stress may lead to the production of cytokines and chemokines, including tumor necrosis factor (TNF)- $\alpha$, transforming growth factor (TGF)- $\beta 1$ and reactive oxygen species (ROS), which are hypothesized to serve a role in the development of $\operatorname{ALD}(1,2)$.

Aldose reductase (AR; additionally termed AKR1B or EC1.1.1.21) catalyzes the reduction of glucose to sorbitol with the aid of the co-factor reduced nicotinamide adenine dinucleotide phosphate, in the polyol pathway (3). The role of $\mathrm{AR}$ in the development of complications in diabetes is well-established (4). Additionally, hepatic AR expression has been observed to be induced in a number of liver disease conditions, including alcoholic liver disease, non-alcoholic fatty liver disease, hepatitis, cirrhosis and hepatocellular carcinoma (5-8). It was previously reported that overexpression of AR induced the production of ROS and TNF- $\alpha$ in liver cells, and that administration of the AR inhibitor zopolrestat attenuated methionine-choline-deficient diet-induced hepatosteatosis and liver inflammation $(8,9)$. However, the role of AR in the development of ALD remains unknown. Therefore, the aim of the present study was to investigate the effect of AR inhibition on ethanol-induced hepatic steatosis and injury in mice and AML12 mouse liver cells, and to investigate the mechanism through which AR affects the development of ALD. 


\section{Materials and methods}

Animal experiments. Male Kunming mice (age, 6 weeks; weight, 22-25 g; total $n=40$ ) were obtained from the Shanghai SLAC Laboratory Animal Co., Ltd. (Shanghai, China) and 5 mice/cage were maintained under a 12-h light/dark schedule, with a temperature range of 20 to $24^{\circ} \mathrm{C}$. Experiments were conducted according to protocols and guidelines approved by the Longyan University Institutional Animal Care and Use Committee (Longyan, China). Liquid diets were based on the modified Lieber-DeCarli formulation and provided 1 $\mathrm{kcal} / \mathrm{ml}$ (prepared by Trophic Animal Feed High-tech Co., Ltd., Nantong, China). The ethanol diet consisted of $18 \%$ of the total energy as protein, $35 \%$ as fat, $19 \%$ as carbohydrate and $28 \%$ as ethanol. In the control diet, ethanol was replaced isocalorically with carbohydrate. Following a 1 -week period of adaptation to the environment with free access to the liquid diet, mice were randomly divided into four experimental groups $(n=10$ mice/group): Control diet-fed mice; control diet-fed mice + zopolrestat (zopol); ethanol diet-fed mice and ethanol diet-fed mice + zopol. Mice were administered $50 \mathrm{mg} / \mathrm{kg} /$ day zopol as a single daily intraperitoneal injection for 4 weeks. Equivalent volumes of saline were administered to the control groups of mice. On the final day, mice were sacrificed under anesthesia. Tissues were snap-frozen in liquid nitrogen immediately following resection and stored at $-80^{\circ} \mathrm{C}$ until further analysis.

Histological examination. Liver tissues were fixed in $10 \%$ formalin for $24 \mathrm{~h}$ at room temperature and were routinely embedded in paraffin. Briefly, tissues were rinsed in tap water for $5 \mathrm{~min}$ at room temperature prior to dehydration with a series of alcohol (70, 80 and 90\%, respectively, for 5 min each, followed by 3 incubations with $100 \%$ alcohol for 5 min each at room temperature). The tissues were then treated twice with xylene for $5 \mathrm{~min}$ each at room temperature, prior to paraffin embedding. Sections $(5-\mu \mathrm{m})$ were deparaffinized in xylene twice for 5 min each and treated with $100 \%$ alcohol twice for $3 \mathrm{~min}$, then 95, 70 and 50\% alcohol, respectively, for $3 \mathrm{~min}$ each at room temperature. Sections were stained with hematoxylin and eosin (H\&E) for histological analysis. Hepatic steatosis was graded as previously described (10), according to the percentage of lipid-laden hepatocytes: $0,0 \% ; 1,0-25 \% ; 2$, $26-50 \% ; 3,51-75 \%$ and $4,76-100 \%$.

Cell culture. AML12 mouse hepatocyte cells were obtained from the American Type Culture Collection (Manassas, VA, USA) and cultured in Dulbecco's modified Eagle's medium/Ham's F12 containing $100 \mathrm{U} / \mathrm{ml}$ penicillin, $100 \mu \mathrm{g} / \mathrm{ml}$ streptomycin and $10 \%$ fetal bovine serum (Hyclone; GE Healthcare Life Sciences, Logan, UT, USA), in a $37^{\circ} \mathrm{C}$ incubator with $5 \% \mathrm{CO}_{2}$.

Detection of ROS generation in AML12 cells. AML12 cells were plated on 24 -well plates at a density of $\sim 1 \times 10^{5}$ cells/well. Following $24 \mathrm{~h}$ of incubation, cells were exposed to $100 \mathrm{mM}$ ethanol and co-treated with $50 \mu \mathrm{M}$ zopol. A total of $36 \mathrm{~h}$ subsequent to ethanol and zopol treatment, ROS generation in cells was determined using ROS assay kit (cat no S0033; Beyotime Institute of Biotechnology, Haimen, China), according to the manufacturer's instructions.
Tissue and cell lipid peroxidation assays. Total lipoperoxides were measured as thiobarbituric acid reactive substances (TBARS) in liver homogenates or cell lysates, using a lipid peroxidation malondialdehyde (MDA) assay kit (cat no S0131; Beyotime Institute of Biotechnology), according to the manufacturer's instructions. TBARS were quantified using MDA as a standard and Microsoft Excel 2013 (Microsoft Corporation. Redmond, WA, USA).

Quantitative analysis of $m R N A$ expression using reverse transcription-quantitative polymerase chain reaction ( $R T-q P C R)$. Total RNA was isolated from tissues and cells using TRIpure reagent (Roche Applied Science, Mannheim, Germany), according to the manufacturer's protocol. cDNA was synthesized from mRNA using RevertAid First Strand cDNA Synthesis kits (Thermo Fisher Scientific, Inc., Waltham, MA, USA). Expression levels of hepatic TNF- $\alpha$, TGF- $\beta 1$, interleukin (IL)-6 and sterol regulatory element binding protein (SREBP)-1c were analyzed. The specific primers used were: Forward, 5'-CGTGCTCCTCACCCACAC-3' and reverse, 5'-GGGTTCATACCAGGGTTTGA-3' for TNF- $\alpha$; forward, 5'-ACAACCACGGCCTTCCCTACTT-3' and reverse, 5'-GTG TAATTAAGCCTCCGACT-3' for IL-6; forward, 5'-CAA CTTCTGTCTGGGACCCT-3' and reverse, 5'-TAGTAGACG ATGGGCAGTGG-3' for TGF- $\beta 1$; forward, 5 '-ATCGGCGCG GAAGCTGTCGGGGTAGCGTC-3' and reverse, 5'-ACTGTC TTGGTTGTTGATGAGCTGGAGCAT-3' for SREBP-1c and forward, 5'-CTATTGGCAACGAGCGGTTCC-3' and reverse, 5'-GCACTGTGTTGGCATAGAGGTC-3' for $\beta$-actin. qPCR was performed using the FastStart Universal SYBR-Green Master Mix (Roche Applied Science). The reaction was performed with the following thermocycling conditions: $95^{\circ} \mathrm{C}$ for $10 \mathrm{~min}$, followed by 35 cycles of $95^{\circ} \mathrm{C}$ for $15 \mathrm{sec}, 53-59^{\circ} \mathrm{C}$ for $30 \mathrm{sec}$ and $72^{\circ} \mathrm{C}$ for $30 \mathrm{sec}$, then a final extension of $72^{\circ} \mathrm{C}$ for $10 \mathrm{~min}$. The ${ }^{2-\Delta \Delta \mathrm{Cq}}$ method (11) was used for quantification and the data were normalized to $\beta$-actin.

Western blot analysis. Tissues and cells were homogenized in ice-cold radioimmunoprecipitation assay buffer (Beyotime Institute of Biotechnology). Protein concentrations of the extracts were measured using a bicinchoninic acid protein assay kit (Beyotime Institute of Biotechnology), according to the manufacturer's protocol. A total of $40 \mu \mathrm{g}$ of each protein sample was loaded and separated using 10-12\% SDS-PAGE and transferred to polyvinylidene difluoride membranes (EMD Millipore, Billerica, MA, USA). Membranes were blotted with $5 \%$ non-fat milk in TBS containing $0.1 \%$ Tween-20 for $1 \mathrm{~h}$ at room temperature. Membranes were then incubated with anti-AR (cat no. sc-17735; 1:500; Santa Cruz Biotechnology, Inc., Dallas, TX, USA), anti-cytochrome P450 2E1 (cat no. ab28146; 1:1,000; CYP2E1; Abcam, Cambridge, UK), anti-phosphorylated AMPK (cat no. 2535; 1:1,000), anti-AMPK (cat no. 2532; 1:1,000) (both from Cell Signaling Technology), anti-GAPDH (cat no. G8795; 1:2,000) or anti- $\beta$-actin (cat no. A2228; 1:2,000) (Sigma-Aldrich; Merck $\mathrm{KGaA}$, Darmstadt, Germany) at $4^{\circ} \mathrm{C}$ overnight, and subsequently with horseradish peroxidase-conjugated anti-goat or anti-rabbit immunoglobulin G (cat nos. AP307P and AB324P, respectively; 1:2,000; Sigma-Aldrich; Merck KGaA) for $2 \mathrm{~h}$ at room temperature. Detection was achieved using the 
SuperSignal Chemiluminescent Substrate kit (Beyotime Institute of Biotechnology).

Statistical analysis. All data were processed and analyzed using GraphPad Prism software, version 5.0 (GraphPad Software, Inc., La Jolla, CA, USA) and were expressed as the mean \pm standard error of the mean. One-way analysis of variance and the post hoc Newman-Keuls multiple comparison test was used for multi-group analyses. $\mathrm{P}<0.05$ was considered to indicate a statistically significant difference.

\section{Results}

$A R$ is induced in the livers of ethanol diet-fed mice and in ethanol-treated mouse AML12 liver cells, and inhibition of $A R$ activity attenuates ethanol-induced hepatic steatosis. Previous studies demonstrated that feeding C57BL/6 mice with Lieber-DeCarli ethanol diets induced fatty liver within 4-8 weeks (12). In order to investigate whether AR was involved in the development of ethanol diet-induced hepatosteatosis, the protein expression levels of hepatic AR were analyzed in mice fed with the ethanol diet. As presented in Fig. 1A, hepatic AR protein expression in C57BL/6 mice fed with the ethanol diet for 4 weeks was increased, compared with mice fed with the control diet. In addition to the induction of AR expression in mouse liver tissue, an increase in AR protein expression was observed in hepatocytes exposed to $100 \mathrm{mM}$ ethanol for 36 h (Fig. 1B).

In order to further determine the role of AR in the development of ethanol-induced hepatosteatosis, AR activity was inhibited by treating mice with an AR-specific inhibitor, zopol. As presented in Fig. 2A and B, examination of H\&E-stained sections demonstrated marked hepatic steatosis in mice fed with the ethanol diet for 4 weeks, while mice fed with the control diet exhibited low-grade steatosis. Following inhibition of AR activity, hepatic steatosis in mice fed with the ethanol diet was significantly attenuated $(\mathrm{P}<0.05)$.

$A R$ inhibition decreases ethanol-induced hepatic oxidative stress in vivo and in vitro. In order to clarify the mechanism through which AR is associated with the development of ethanol-induced steatosis, the effect of AR inhibition on hepatic lipoperoxide production was investigated. As presented in Fig. 3A, consumption of the ethanol diet resulted in an increase in hepatic TBARS levels, compared with the control diet, and the increase was attenuated significantly by treating the ethanol diet-fed mice with zopol $(\mathrm{P}<0.001)$. The protein expression of CYP2E1, a principal mediator of lipid peroxidation $(13,14)$, was induced by ethanol, and AR inhibition significantly prevented the induction of CYP2E1 (Fig. 3B). In addition, the protective effect of AR inhibition on ethanol-induced oxidative stress was analyzed in vitro. As presented in Fig. $3 \mathrm{C}$ and D, inhibition of AR resulted in a 33.0\% decrease in MDA levels $(\mathrm{P}<0.001)$ and a $33.9 \%$ decrease in ROS levels $(\mathrm{P}<0.05)$ in ethanol-treated AML12 cells. However, CYP2E1 protein expression was not detected in AML12 cells. The results of the present study demonstrated that the induction of AR by ethanol may promote hepatic oxidative stress in mice with alcoholic liver disease, and that the increased hepatic oxidative stress is due in part to the AR-mediated induction of CYP2E1.
A

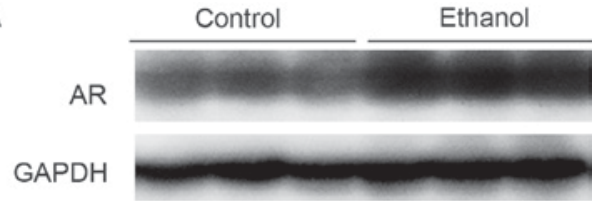

B

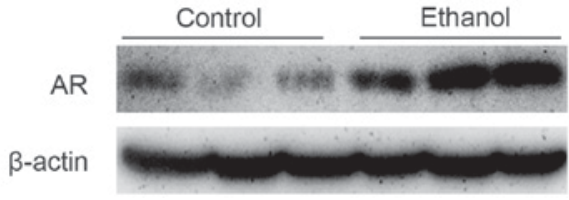

Figure 1. Hepatic AR is induced by ethanol in vivo and in vitro. Representative western blot analyses demonstrate the induction of hepatic AR protein expression in (A) ethanol-fed C57BL/6 mice and (B) ethanol-treated AML12 cells. AR, aldose reductase

AR inhibition activates AMPK and suppresses SREBP-1c $m R N A$ expression. Previous studies demonstrated that AMPK inactivation was associated with the development of ethanol-induced hepatic steatosis $(15,16)$. In order to clarify the mechanisms whereby AR affects lipid accumulation in hepatocytes, the effect of AR inhibition on AMPK activity was investigated. As presented in Fig. 4A, phosphorylated AMPK protein expression levels in the livers of ethanol-fed mice treated with zopol were increased compared with ethanol-fed mice, indicating that AR inhibition activated hepatic AMPK in ethanol-fed mice. The activation on AMPK by the AR inhibitor was additionally confirmed in ethanol-treated AML12 cells (Fig. 4B). The effect of AR inhibition on the mRNA expression of SREBP-1c, a transcriptional factor regulated by AMPK which controls the synthesis of fatty acids, was assessed in the livers of ethanol-fed mice. As presented in Fig. 4C, AR inhibition did not significantly decrease the mRNA expression levels of SREBP-1c in ethanol-fed mice when compared with the ethanol-fed group. However, AR inhibition significantly decreased the mRNA expression levels of SREBP-1c in ethanol-treated AML12 cells (Fig. 4D).

AR inhibition suppresses the mRNA expression of certain inflammatory mediators in the livers of ethanol diet-fed mice and in hepatocytes. In order to evaluate the effect of AR inhibition on the development of ethanol-induced liver injuries, the expression levels of certain proinflammatory cytokines were assessed, including TNF- $\alpha$, IL- 6 and TGF- $\beta 1$. Mice fed on the ethanol diet exhibited a marked elevation of hepatic mRNA expression of TNF- $\alpha$ compared with mice fed on the control diet (Fig. 5A). However, the ethanol-induced TNF- $\alpha$ mRNA elevation was significantly attenuated by $51.4 \%(\mathrm{P}<0.01)$ in mice treated with zopol. Compared with TNF- $\alpha$, the effect of AR inhibition on IL- 6 and TGF- $\beta 1$ was less significant. Consistent with the in vivo results, AR inhibition in ethanol-treated AML12 cells resulted in a significant decrease in the mRNA expression levels of TNF- $\alpha(\mathrm{P}<0.01$; Fig. 5B). AR inhibition in ethanol-treated AML12 cells resulted in a significant decrease in the mRNA expression levels of IL-6 $(\mathrm{P}<0.05)$ and TGF- $\beta 1$ $(\mathrm{P}<0.05)$. The results of the present study demonstrated that hepatic AR elevation in mice fed on the ethanol diet may directly affect the production of pro-inflammatory cytokines, particularly TNF- $\alpha$, in the liver. 

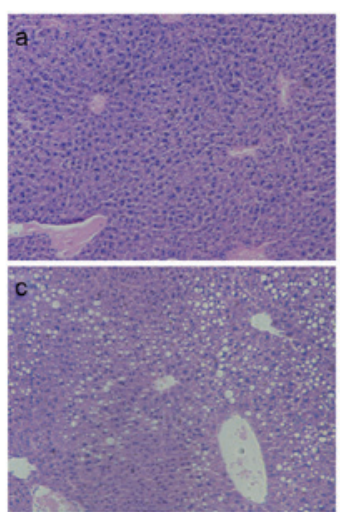
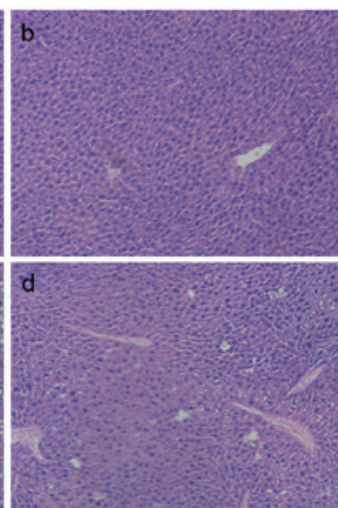

B

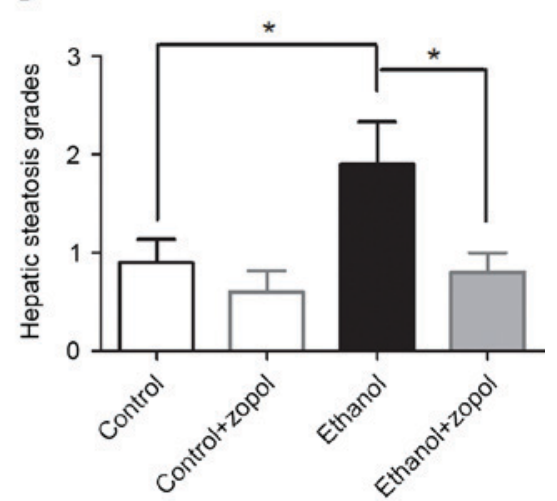

Figure 2. Treatment with an AR inhibitor improves ethanol-induced steatosis in C57BL/6 mice. (A) Hematoxylin and eosin-stained liver sections from mice fed with: (a) Control diet; (b) control diet + zopol; (c) ethanol diet; and (d) ethanol diet + zopol. Slides are representative of eight separate experiments (magnification, $\mathrm{x} 100)$. (B) Effect of AR inhibition on scores for hepatic steatosis $(\mathrm{n}=8)$. Values are expressed as the mean \pm standard error of the mean. ${ }^{*} \mathrm{P}<0.05$. AR, aldose reductase; zopol, zopolrestat.
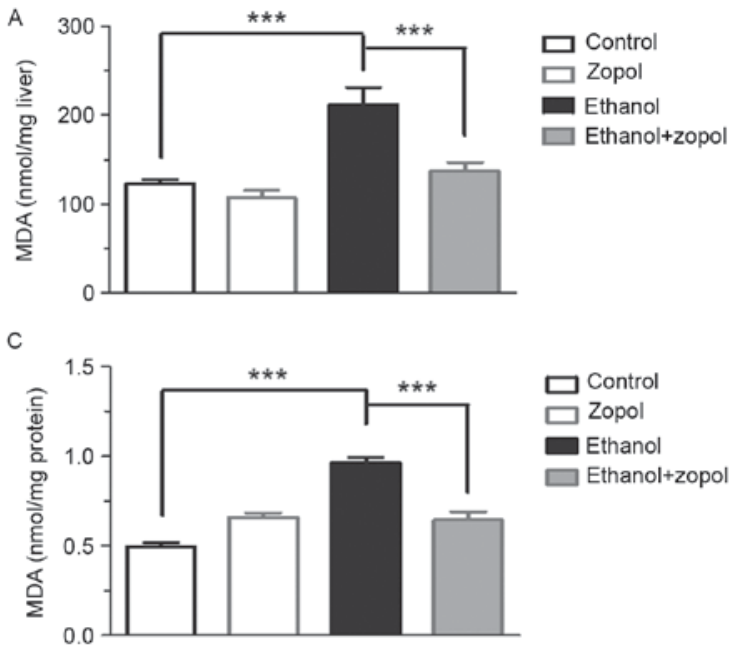

B
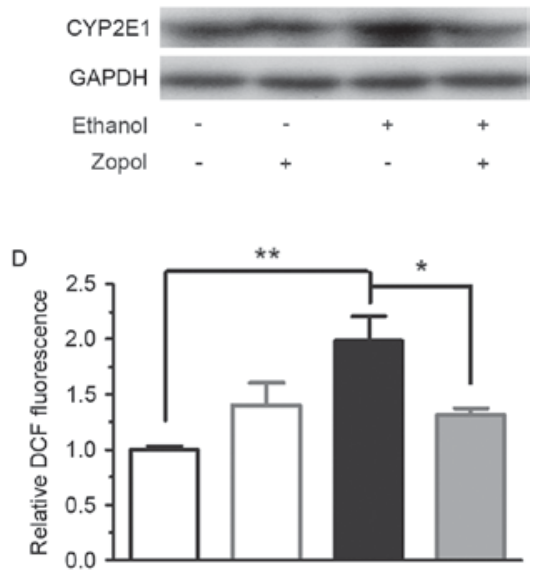

Figure 3. Inhibition of AR attenuates hepatic lipoperoxide content and CYP2E1 expression in ethanol-fed C57BL/6 mice, and attenuates lipoperoxide content and ROS levels in AML12 cells. (A) Hepatic lipoperoxide content and (B) CYP2E1 protein expression were determined in mice fed with the control diet, ethanol diet or ethanol diet treated with zopol for 4 weeks $(n=4)$. (C) Lipoperoxide content and (D) ROS levels were assayed in ethanol-treated AML12 cells treated with zopol for $36 \mathrm{~h}(\mathrm{n}=4)$. Values are expressed as the mean \pm standard error of the mean. ${ }^{* * *} \mathrm{P}<0.001 ;{ }^{* *} \mathrm{P}<0.01 ;{ }^{*} \mathrm{P}<0.05$. AR, aldose reductase; CYP2E1, cytochrome P450 2E1; ROS, reactive oxygen species; zopol, zopolrestat; DCF, dichlorofluorescein; MDA, malondialdehyde.

\section{Discussion}

The induction of AR expression has been observed in certain liver disease conditions, including alcoholic liver disease and non-alcoholic fatty liver disease $(6,8)$. Inhibition of AR improves the development of hepatic steatosis and liver inflammation in mice with non-alcoholic steatohepatitis (7-9). However, the role of AR in the development of ALD remains unclear. The results of the present study demonstrated that hepatic AR protein was induced in ethanol diet-induced hepatosteatosis in C57BL/6 mice, and in ethanol-treated mouse AML12 liver cells. Pharmacological inhibition of AR was conducted in the ethanol diet-fed C57BL/6 mice, to examine the role of AR in the development of ethanol-induced hepatosteatosis. It was demonstrated that AR inhibition improved the development of hepatosteatosis. The results of the present study indicated that AR may be involved in the development of ALD.
The alcohol metabolite acetaldehyde has previously been demonstrated to be one of the important causes of hepatic triglyceride accumulation and hepatocellular injury in alcoholic fatty livers (17). AR is able to catalyze the reduction of a variety of aldehydes and carbonyls $(18,19)$. Therefore, AR has been postulated to serve a cytoprotective function by rapidly detoxifying aldehydes. AR inhibitors have been reported to prevent injuries in a variety of rodent models, including ischemia/reperfusion-induced liver (20), arterial balloon (21) and ischemic myocardial injuries (22). In the present study, it was demonstrated that AR exacerbated hepatocyte injury. As AR possesses the capacity to rapidly detoxify aldehydes, it is notable that AR inhibition prevented hepatocyte injury. The present study demonstrated that $\mathrm{AR}$ inhibition prevented the induction of CYP2E1 in ethanol diet-fed C57BL/6 mice. CYP2E1 serves an important role in the pathogenesis of ALD $(13,14,23)$. CYP2E1 is able to catalyze the reduction of alcohol to acetaldehyde in the liver, resulting in the generation 
A

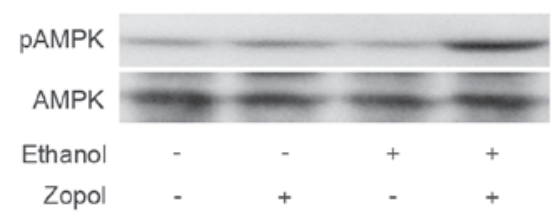

C

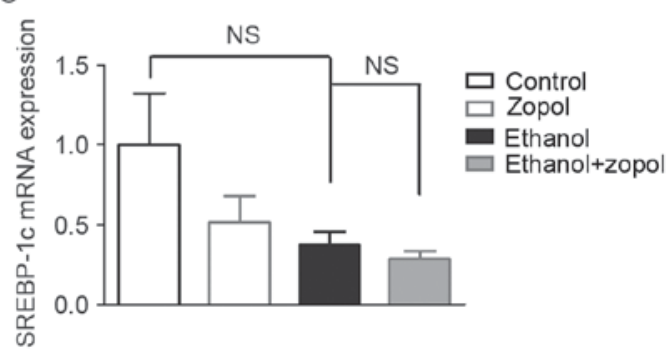

B

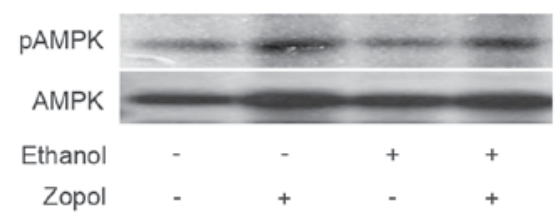

D

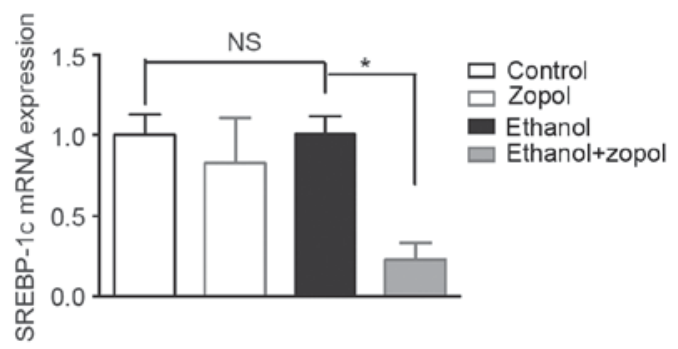

Figure 4. Effect of AR inhibition on the activation of AMPK and the mRNA expression of SREBP-1c. Activation of AMPK in (A) zopol-treated mice fed with ethanol diet and (B) zopol-treated AML12 liver cells exposed to ethanol was determined using western blotting. mRNA expression of SREBP-1c in (C) zopol-treated mice fed with ethanol diet and (D) zopol-treated AML12 liver cells exposed to ethanol was analyzed using reverse transcription-quantitative polymerase chain reaction, standardized against an internal control ( $\beta$-actin). Data are expressed as fold differences compared with values obtained in control cells $(n=4)$. Values are expressed as the mean \pm standard error of the mean. $\mathrm{P}<0.05$. NS, not significant; SREBP-1c, sterol regulatory element binding protein-1c; AR, aldose reductase; AMPK, 5'-AMP-activated protein kinase; zopol, zopolrestat; p, phosphorylated.

A

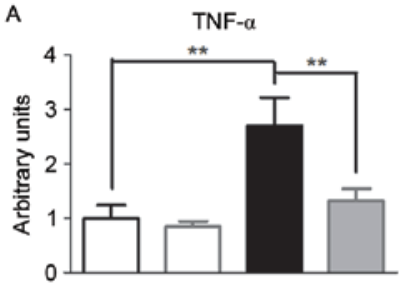

B

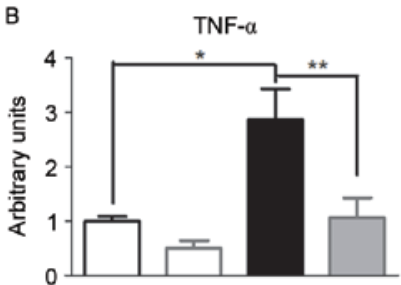

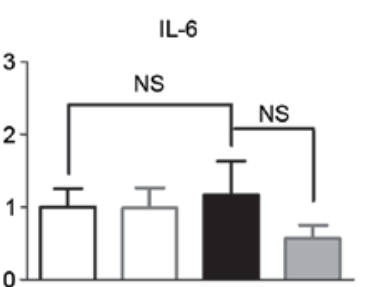

IL-6

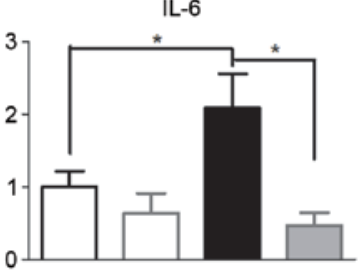

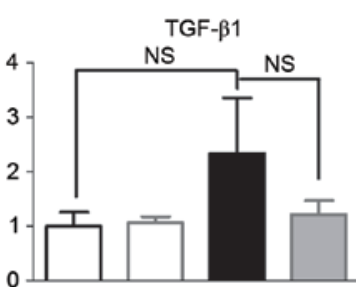

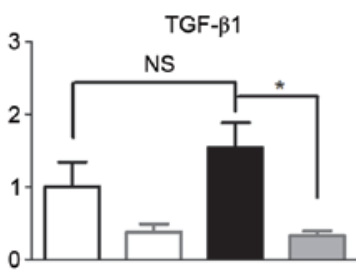

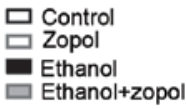

Control

므 Zopol

Ethanol

Ethanol+zopol

Figure 5. AR inhibition attenuates the ethanol-induced TNF- $\alpha$ mRNA expression in C57BL/6 mice and AML12 cells. mRNA expression levels of TNF- $\alpha$, IL-6 and TGF- $\beta 1$ were analyzed in (A) C57BL/6 mice and (B) AML12 cells using reverse transcription-quantitative polymerase chain reaction, standardized against an internal control ( $\beta$-actin). Data are expressed as fold differences compared with values obtained in control cells $(\mathrm{n}=4)$. Values are expressed as the mean \pm standard error of the mean. ${ }^{* *} \mathrm{P}<0.01$; " $\mathrm{P}<0.05$. NS, not significant; AR, aldose reductase; TNF, tumor necrosis factor; IL, interleukin; TGF, transforming growth factor; zopol, zopolrestat.

of ROS, including the superoxide anion, hydrogen peroxide and the hydroxyl radical. The generation of ROS may initiate lipid peroxidation in the liver (13). The results of the present study suggested that the elevation of AR resulted in the induction of hepatic CYP2E1 expression in mice with ALD and, consequently, exacerbated lipid peroxidation and oxidative stress, and affected the development of ALD. Therefore, the prevention of the induction of hepatic CYP2E1 in mice with ALD may be part of the mechanism via which AR inhibition attenuates lipid peroxidation and ameliorates ALD.

AMPK is an enzyme that serves a role in cellular energy homeostasis. The effect of AMPK inhibition is suppression of fatty acid oxidation and stimulation of lipogenesis (24). Ethanol has been reported to suppress hepatic AMPK activity, and the inhibition of AMPK by ethanol serves an important role in the development of hepatosteatosis induced by chronic alcohol consumption $(15,16)$. In the present study, it was demonstrated that AR inhibition activated AMPK in ethanol-treated AML12 liver cells and in the livers of mice fed with ethanol. Therefore, activation of hepatic AMPK may be part of the mechanism via which AR inhibition ameliorates ethanol-induced hepatosteatosis.

It is well-known that chronic alcohol consumption increases TNF- $\alpha$ production and leads to hepatosteatosis and liver injury (2). Studies have demonstrated that the expression of TNF- $\alpha$ is elevated in patients with alcoholic liver diseases $(25,26)$. In addition, TNF- $\alpha$ has been observed to induce lipid accumulation, and result in apoptosis and inflammation in liver cells (27). Therefore, the improvement of steatosis and liver injury in alcoholic livers by treatment with an AR inhibitor may be partially attributed to an inhibitory effect on ethanol-induced TNF- $\alpha$ mRNA overexpression. 
In conclusion, the present study demonstrated that AR inhibition significantly ameliorated alcohol-induced hepatic steatosis in mouse AML12 liver cells and in Kunming mice, potentially by activating AMPK, decreasing CYP2E1-mediated oxidative stress and suppressing the expression of pro-inflammatory cytokines. These results indicate that AR inhibitors may have potential as alternative therapeutic strategies for the treatment of alcoholic fatty liver diseases.

\section{Acknowledgements}

The present study was supported in part by the Training Program of Fujian Excellent Talents in University (grant no. MJR201558) and the Science Planning Program of Longyan University (grant no. LG2014012).

\section{References}

1. Ishii H, Kurose I and Kato S: Pathogenesis of alcoholic liver disease with particular emphasis on oxidative stress. J Gastroenterol Hepatol 12: S272-S282, 1997.

2. Kawaratani H, Tsujimoto T, Douhara A, Takaya H, Moriya K, Namisaki T, Noguchi R, Yoshiji H, Fujimoto M and Fukui H: The effect of inflammatory cytokines in alcoholic liver disease. Mediators Inflamm 2013: 495156, 2013.

3. Hers HG: Aldose reductase. Biochim Biophys Acta 37: 120-126, 1960 (In French).

4. Nishimura-Yabe C: Aldose reductase in the polyol pathway: A potential target for the therapeutic intervention of diabetic complications. Nihon Yakurigaku Zasshi 111: 137-145, 1998 (In Japanese).

5. Brown KE, Broadhurst KA, Mathahs MM, Kladney RD, Fimmel CJ, Srivastava SK and Brunt EM: Immunodetection of aldose reductase in normal and diseased human liver. Histol Histopathol 20: 429-436, 2005.

6. O'Connor T, Ireland LS, Harrison DJ and Hayes JD: Major differences exist in the function and tissue-specific expression of human aflatoxin B1 aldehyde reductase and the principal human aldo-keto reductase AKR1 family members. Biochem J 343: 487-504, 1999

7. Qiu L, Lin J, Xu F, Gao Y, Zhang C, Liu Y, Luo Y and Yang JY: Inhibition of aldose reductase activates hepatic peroxisome proliferator-activated receptor- $\alpha$ and ameliorates hepatosteatosis in diabetic db/db mice. Exp Diabetes Res 2012: 789730, 2012.

8. Qiu L, Lin J, Ying M, Chen W, Yang J, Deng T, Chen J, Shi D and Yang JY: Aldose reductase is involved in the development of murine diet-induced nonalcoholic steatohepatitis. PLoS One 8: e73591, 2013.

9. Chen T, Shi D, Chen J, Yang Y, Qiu M, Wang W and Qiu L: Inhibition of aldose reductase ameliorates diet-induced nonalcoholic steatohepatitis in mice via modulating the phosphorylation of hepatic peroxisome proliferator-activated receptor $\alpha$. Mol Med Rep 11: 303-308, 2015.

10. Peng Z, Borea PA, Varani K, Wilder T, Yee H, Chiriboga L, Blackburn MR, Azzena G, Resta G and Cronstein BN: Adenosine signaling contributes to ethanol-induced fatty liver in mice. J Clin Invest 119: 582-594, 2009.
11. Livak KJ and Schmittgen TD: Analysis of relative gene expression data using real-time quantitative PCR and the 2(-Delta Delta C(T)) method. Methods 25: 402-408, 2001.

12. Lieber CS, DeCarli LM and Sorrell MF: Experimental methods of ethanol administration. Hepatology 10: 501-510, 1989.

13. Leung TM and Nieto N: CYP2E1 and oxidant stress in alcoholic and non-alcoholic fatty liver disease. J Hepatol 58: 395-398, 2013.

14. Lu Y and Cederbaum AI: CYP2E1 and oxidative liver injury by alcohol. Free Radic Biol Med 44: 723-738, 2008.

15. You M, Matsumoto M, Pacold CM, Cho WK and Crabb DW: The role of AMP-activated protein kinase in the action of ethanol in the liver. Gastroenterology 127: 1798-1808, 2004.

16. Sid B, Verrax J and Calderon PB: Role of AMPK activation in oxidative cell damage: Implications for alcohol-induced liver disease. Biochem Pharmacol 86: 200-209, 2013.

17. Barry RE: Role of acetaldehyde in the pathogenesis of alcoholic liver disease. Br J Addict 83: 1381-1386, 1988.

18. Vander Jagt DL and Hunsaker LA: Substrate specificity of reduced and oxidized forms of human aldose reductase. Adv Exp Med Biol 328: 279-288, 1993.

19. Vander Jagt DL, Kolb NS, Vander Jagt TJ, Chino J, Martinez FJ, Hunsaker LA and Royer RE: Substrate specificity of human aldose reductase: Identification of 4-hydroxynonenal as an endogenous substrate. Biochim Biophys Acta 1249: 117-126, 1995.

20. Zhang C, Huang C, Tian Y and Li X: Polyol pathway exacerbated ischemia/reperfusion-induced injury in steatotic liver. Oxid Med Cell Longev 2014: 963629, 2014.

21. Ruef J, Liu SQ, Bode C, Tocchi M, Srivastava S, Runge MS and Bhatnagar A: Involvement of aldose reductase in vascular smooth muscle cell growth and lesion formation after arterial injury. Arterioscler Thromb Vasc Biol 20: 1745-1752, 2000.

22. Tracey WR, Magee WP, Ellery CA, MacAndrew JT, Smith AH, Knight DR and Oates PJ: Aldose reductase inhibition alone or combined with an adenosine A (3) agonist reduces ischemic myocardial injury. Am J Physiol Heart Circ Physiol 279: H1447-H1452, 2000.

23. Morimoto M, Hagbjörk AL, Nanji AA, Ingelman-Sundberg M, Lindros KO, Fu PC, Albano E and French SW: Role of cytochrome P4502E1 in alcoholic liver disease pathogenesis. Alcohol 10: 459-464, 1993.

24. Viollet B, Guigas B, Leclerc J, Hébrard S, Lantier L, Mounier R, Andreelli $\mathrm{F}$ and Foretz M: AMP-activated protein kinase in the regulation of hepatic energy metabolism: From physiology to therapeutic perspectives. Acta Physiol (Oxf) 196: 81-98, 2009.

25. An L, Wang $X$ and Cederbaum AI: Cytokines in alcoholic liver disease. Arch Toxicol 86: 1337-1348, 2012.

26. McClain CJ and Cohen DA: Increased tumor necrosis factor production by monocytes in alcoholic hepatitis. Hepatology 9: 349-351, 1989 .

27. Endo M, Masaki T, Seike M and Yoshimatsu H: TNF-alpha induces hepatic steatosis in mice by enhancing gene expression of sterol regulatory element binding protein-1c (SREBP-1c). Exp Biol Med (Maywood) 232: 614-621, 2007. 